\title{
RÉSULTATS SUR LA REPRODUCTION DE ACIPENSER BAERI EN 1982
}

\author{
P. WILLIOT, R. BRUN(*)
}

(*) CEMAGREF - Division Aménagements Littoraux et Aquaculture, B.P. 3, 33610 CESTAS PRINCIPAL

\section{RÉSUMÉ}

La reproduction a eu lieu fin janvier $1982 ; 8$ femelles pesant de 4 à $6 \mathrm{~kg}$ et 9 mâles ont été sélectionnés.

La spermiation et l'ovulation ont été obtenues par injection d'hypophyse de carpe ( $2 \mathrm{mg} / \mathrm{kg}$ pour les mâles, $4 \mathrm{mg} / \mathrm{kg}$ pour les femelles).

Seulement 4 femelles ont donné des ovules et uniquement par pression abdominale. Le traitement anticoagulant des œufs a été réalisé par brassage dans une suspension d'argile.

L'incubation a eu lieu en bouteille de Zoug et pour une température d'eau de $12^{\circ} \mathrm{C}$. L'éclosion est intervenue pour environ $110^{\circ} \mathrm{C}$.jour. femelle.

Le nombre de larves est égal à 1800 , dont la majeure partie provient d'une seule vivantes.

Parmi les 4 femelles ayant subi une césarienne en 1981, 3 d'entre elles sont

\section{SUMMARY}

Reproduction of Acipenser baeri was experimented at the end of January 1982 with 8 females weighing 4 to $6 \mathrm{~kg}$ and 9 males which had been selected.

Spermiation and ovulation were obtained by injection of carp pituitary $(2 \mathrm{mg} / \mathrm{kg}$ into males and $4 \mathrm{mg} / \mathrm{kg}$ into females).

Only 4 females produced ova and only by stripping.

A clay suspension was used to prevent the eggs from sticking together.

Incubation was made in Zug jars at a water temperature of $12^{\circ} \mathrm{C}$. Hatching occured after about $110^{\circ} \mathrm{C}$ day. The quantity of larvae obtained was 1800 , most of them from one female.

Among the 4 females which had had a caesarian operation in 1981 three of them are still alive.

La reproduction artificielle de l'esturgeon Acipenser baeri réalisée en 1981 a donné des résultats encourageants. Cette technique a été à nouveau mise en ceuvre cette année sur des bases identiques et dans la même écloserie.

\section{GESTION ET SÉLECTION DES GENITEURS}

Deux lots d'animaux ont constitué le stock de géniteurs utilisés pour la reproduction 1982.

- 1er lot: animaux stockés en Brenne et dans le même étang depuis octobre 1980; parmi ceux-ci 12 individus ( 7 mâles et 5 femelles) avaient été utilisés pour la reproduction en 1981 ; les autres ne présentaient pas une maturité sexuelle suffisante. 
- 2ème lot: animaux stockés dans la pisciculture de l'INRA à Donzacq et transportés en Brenne dans le même étang que les géniteurs du premier lot à la suite d'un tri effectué en octobre 1981 selon l'état de maturation des gonades observé en pratiquant les biopsies. A cette date, de nombreuses femelles ont déjà des ovocytes très développés, diamètre compris entre 2 et $2,5 \mathrm{~mm}$. Pour certaines d'entre elles qui ont les ovocytes gris foncé, souples avec une membrane externe lisse et la vésicule germinative en périphérie, une tentative de reproduction devrait être immédiate. Pour d'autres, les ovocytes présentent des signes vraisemblables de surmaturité ; ils sont gris clair, mous, avec souvent une enveloppe ridée.

De nombreux mâles sont également dans un état de maturité avancée, une légère incision des gonades entraîne un écoulement du sperme. moment là.

Le manque d'installations sur place n'a pas permis de tenter une reproduction à ce

Ainsi, comme l'année précédente pour essayer de freiner, voire d'arrêter la gamétogenèse, les animaux jugés aptes à la reproduction ont été stockés en Brenne le 3.11.81.

L'ensemble du stock a été pêché le 14 janvier 1982.

Au moment de la pêche on constate :

- une diminution relativement importante $(24 \%)$ du nombre d'animaux en étang depuis plus d'un an:

- une bonne survie des femelles ayant subi une laparotomie lors de la dernière reproduction ( 3 sur 4 vivantes) mais elles sont maigres.

Sur l'ensemble des animaux on a contrôlé le poids et la maturité sexuelle.

A la suite des observations réalisées 17 géniteurs ont été retenus:

- 9 mâles

- 8 femelles dont 4 seulement semblaient être au stade physiologique optimal.

Tous les mâles sélectionnés et 6 femelles sur 8 provenaient du stock récemment transféré de Donzacq.

Depuis la pêche jusqu'à leur entrée dans l'écloserie le 21 janvier, soit environ 8 jours, les animaux ont été maintenus dans une eau à $8-9^{\circ} \mathrm{C}$.

Ensuite, cette température a été portée à $9-10^{\circ} \mathrm{C}$ durant 5 jours, puis à $12^{\circ} \mathrm{C}$ pendant 2 jours.

\section{REPRODUCTION}

\subsection{Stimulation de la spermiation et de l'ovulation}

Eu égard aux indications obtenues l'an dernier, seule l'hypophyse de carpe a été utilisée comme source d'hormone.

Les doses et séquences adoptées sont résumées ci-dessous :

- Mâles : 1 seule injection de $2 \mathrm{mg} / \mathrm{kg}$

- Femelles : Dose totale de $4 \mathrm{mg} / \mathrm{kg}$ répartie de la manière suivante :

- pour 3 animaux $(p=4,33 \pm 0.12 \mathrm{~kg}): 1$ seule injection.

- pour 5 animaux $(p=5,3 \pm 0,9 \mathrm{~kg}): 2$ injections à 13 heures

d'intervalle, la première correspondant à $1 / 10$ de la dose totale.

Le broyat d'hypophyse a été dilué dans du sérum physiologique à 7 pour mille et pour chaque injection la quantité de solution injectée est comprise entre 2 et $2,5 \mathrm{~cm}^{3}$.

Après les injections, la température de l'eau a été d'abord maintenue constante et égale à $12^{\circ} \mathrm{C}$.

Cependanit 24 heures après la première injection, la température a atteint $14^{\circ} \mathrm{C}$ durant $\mathbf{4}$ heures, suite à un mauvais fonctionnement d'une thermovanne.

Ensuite elle a pu être stabilisée à $13^{\circ} \mathrm{C}$.

\subsection{Observations}

Máles : 33 heures après l'injection, l'ensemble des animaux donne plus ou moins de sperme par massage abdominal. 
Mais seulement $50 \%$ d'entre eux en donneront régulièrement durant une trentaine d'heures, chaque prélèvement d'ovules étant précédé d'une collecte de sperme.

Femelles: 4 sur 8 ont donné des ovules 50 heures environ après la première injection quel que soit le fractionnement, ce qui confirme nos résultats antérieurs (WILLIOT, ROUAULT, 1982) et les données de la littérature (KHAKIMOULLIN, 1979).

Cependant, un résultat inattendu a été enregistré lors des manipulations. Nous avons obtenu les ovules uniquement par pression abdominale, c'est-à-dire sans avoir besoin d'ouvrir les femelles.

Plusieurs constations doivent être apportées:

1 - Chez de nombreux animaux, l'oviducte était obstrué par un bouchon dont il était souvent difficile d'obtenir l'expulsion par pression des flancs. Ce bouchon gris blanchâtre était long de $2 \mathrm{~cm}$ environ et avait un diamètre supérieur à $0,5 \mathrm{~cm}$ et était composé en partie de coques d'œufs.

2 - Sur les animaux dont on n'a pas pu obtenir d'ovules par l'orifice génital, on a pratiqué une biopsie de contrôle. Dans tous les cas, les ovocytes restaient accrochés au follicule.

3 - Sur une femelle nous avons pu obtenir des ovules 6 fois sur une période de $17 \mathrm{~h}$. Les trois autres n'ont donné que quelques ovules en une fois.

4 - Plusieurs mesures de poids de 10 ovules humides amènent à estimer qu'il y a 60 ovules/g.

\subsection{Fécondation - Traitement des coufs}

La technique courante est la suivante:

Les ovules sont pesés, en fonction de ce résultat on prend un volume déterminé de sperme que l'on dilue dans de l'eau. Le mélange ainsi obtenu est versé doucement dans la cuvette où se trouvent les ovules.

On brasse doucement l'ensemble avec la main durant environ 5 minutes puis on rince plusieurs fois avant d'effectuer le traitement antiadhésif.

Dans le cas présent, l'apparition de la gangue collante était tellement rapide que :

- nous n'avons pas pesé les ovules, donc la quantité de sperme était approximative :

- il a été nécessaire d'écourter parfois de plus de $50 \%$ le temps de brassage nécessaire ( 2 a 3 minutes au lieu de 5 minutes) à une bonne fécondation:

- le traitement antiagglomérant des cufs réalisé avec de l'argile a dû être effectué 2 fois sur certains lots.

\subsection{Incubation}

Chaque lot d'ovules a été mis dans une bouteille de Zoug. La température de l'eau fut maintenue constante à $12^{\circ} \mathrm{C}$.

Le tout début de l'éclosion est intervenu 10 jours environ après la mise en incubation soit pour 2600 a $2700^{\circ}$ heure ou 107 a $112^{\circ}$ jour.

Pratiquement l'éclosion s'est échelonnée sur plus de 24 heures. Le début de l'éclosion fut simultané pour tous les œufs provenant de la même femelle, bien que 17 heures séparaient les prélèvements extrêmes.

A cause des difficultés du traitement antiagglomérant, tous les lots étaient plus ou moins envahis de champignons.

Un traitement au vert malachite était effectué chaque jour pour limiter leur extension.

Afin d'acclimater les larves à la température de l'eau dans laquelle elles allaient être transférées (soit $16^{\circ} \mathrm{C}$ ) dès le début de l'éclosion on a remonté la température de 12 à $14,5^{\circ} \mathrm{C}$.

Sans avoir la preuve (manque de témoin), il est possible que cela ait eu une influence négative sur les éclosions car on a constaté de nombreux avortements à ce stade.

Dès que le début de l'éclosion intervenait dans une bouteille, son contenu était mis dans une petite cage immergée. 


\section{RESULTATS}

Au total, nous avons eu environ 1800 larves.

$85 \%$ des larves obtenues proviennent d'une seule femelle.

Et pour cette femelle, $73 \%$ proviennent de deux prélèvements d'ovules qui ont eu lieu de 54 à 56 heures après la première injection hypophysaire.

\section{TRAITEMENT DES GÉNITEURS}

Toutes les femelles ayant subi une incision abdominale pour biopsie ont reçu une injection de sulfate de framycétine à raison de $30 \mathrm{mg} / \mathrm{kg}$.

De plus, les plaies ont été désinfectées par aérosol contenant du chloramphenicol et du dichlorophène.

\section{Discussion}

Trois considérations empêchent d'émettre des conclusions précises sur cette expérience qui portait essentiellement sur les doses d'hormones; ce sont :

- une préparation imparfaite des géniteurs:

- un manque de sûreté dans l'appréciation du stade de maturation;

- l'accident dans le contrôle de la température de l'eau après les injections.

Cependant, on peut souligner 3 aspects intéressants de ce travail en comparant aux résultats de 1981 .

1 - L'hypophyse de carpe en tant que stimulant de la spermiation et de l'ovulation est en partie confirmé:

2 - sur une femelle surtout, les ovules ont été obtenus sans opération. Sur cet animal nous $n$ 'avons pas constaté l'existence de bouchon dans l'oviducte. Elle a permis d'obtenir environ 1530 larves; ce nombre comparé à la moyenne de l'an dernier sur 5 femelles correspondrait à $85 \%$ de ce que l'on pouvait attendre.

Bien qu'inférieur, cela reste du même ordre de grandeur et des manipulations plus attentives permettraient sans doute d'améliorer ce résultat.

3 - La survie des femelles opérées en 1981 est a priori intéressante bien qu'aucun soin particulier n'ait été apporté aux conditions d'élevage (alimentation).

$\mathrm{Si}$ ces techniques, obtention des ovules par pression abdominale ou avec césarienne, s'avéraient généralisables, cela permettrait d'envisager des programmes de sélection avec de nouvelles bases.

Enfin, nous nous attacherons à l'avenir à améliorer autant que possible nos connaissances sur la physiologie du stock d'esturgeons sibériens et à poursuivre les premiers essais de grossissement des jeunes.

\section{BIBLIOGRAPHIE}

BILLARD R., 1979. La gamétogenèse, le cycle sexuel et le contrôle de la reproduction chez les poissons téléostéens. Bull. Fr. Pisc., 273, 117-136.

KHAKIMOULLIN A.A., 1979. Durée de maturation de l'esturgeon sibérien après injection hypophysaire. Économie des pêches no 8, 1979. Éd. Industries alimentaires et légères, Économie des Pêches, URSS.

WILLIOT P., CHARLON N., 1978. Rapport sur l'élevage des esturgeons en URSS. Ministère de l'Agriculture, CTGREF-INRA, Janvier 1978.

WILLIOT P., ROUAULT., 1982. Compte rendu d'une première reproduction en France de l'esturgeon sibérien Acipenser baeri. Bull. Fr. Pisc. 286, 255-261. 